\title{
GEODESIC MOTION IN THE 5D MAGNETIZED SCHWARZSCHILD-LIKE SOLUTIONS
}

\author{
Tonatiuh Matos and Nora Bretón \\ Departamento de Física, CINVESTAV del IPN \\ Apartado 14-740, 07000 México, D. F.
}

\begin{abstract}
Geodesics for a 5D magnetized Schwarzschild-like solution are analyzed by reducing the problem to the motion of a test particle in an effective potential. In absence of magnetic field comparison is established with Schwarzschild's geometry. Embedding diagrams are constructed in order to visualize the geometry of the metric. The study performed here is also valid, when the electromagnetic interactions are neglected, for the low energy superstring theory and the Brans-Dicke theory.
\end{abstract}

PACS: 97.60.Lf, 04.20.Me. 


\section{Introduction}

Since many of the astrophysical objects like pulsars, quasars or black holes are endowed with magnetic fields, it is of great interest to study solutions of Einstein's equations which represent massive magnetic multipoles, in particular, magnetic dipole.

However, exact solutions of Einstein's equations interpretable as massive magnetic dipole were found only recently [1] and most of them are rather cumbersome to be studied in analytic form.

On the other hand it is well known that the 5D solutions of Einstein's equations in vacuum when projected into the four dimensional spacetime correspond to solutions of Einstein's equations with an energy-momentum tensor of a gravitational field coupled with an electromagnetic field and also with a massless scalar field [2]. The 5D equations without sources may be reduced to the 4D ones with sources, provided an appropriate definition is made for the energy-momentum tensor of matter in terms of the extra part of the geometry. In relation with this point it is not clear how to interpret any higher dimensional theory physically. Interpretation of the fifth dimension has been done as a massless scalar field which can be or not associated with a fluid density [3]. In other works it has been interpreted as a "magnetic mass" [4]. Also interpretation has been done as a fifth geometric property which shows up near horizon [5]. In any case, it is worth to explore ways of interpreting the properties of the $5 \mathrm{D}$ solutions in a $4 \mathrm{D}$ world.

Recently, solutions were obtained using the Chiral formalism in 5D [6]. These solutions represent bodies endowed with a magnetic field and whose gravitational potential possess a Schwarzschild-like behaviour. They were derived starting with a pseudo-riemannian 5D space which is a gauge invariant model under an U(1) group. The Einstein's equations are solved in the potential space and from these potentials the metric components are determined.

The solution studied in this paper was derived in [7] and is a model for the magnetic dipole of static bodies based in a 5D gauge theory that in $4 \mathrm{D}$ corresponds to a massive magnetic dipole coupled to a massless scalar field. This solution is analytically tracktable so it is worthwhile to ask about the plausibility of such a model compared with Schwarzschild's solution predictions. 
We note that the analysis carried out here is valid, when the electromagnetic field is neglected, for low energy superstring theory ( $\alpha=1$ in Ref. [8]), for Einstein-Maxwell with scalar field ( $\alpha=0$ in Ref. [8]) and for Brans-Dicke theory ( $F=0$ in Ref. [8]).

In the following Section 2 general features of the metric are presented. In Section 3.1, the effective potential and the effect of variation of the magnetic dipole are described. It is seen the effect of the scalar field on the spacetime near $r=2 M$. In Section 3.2, in absence of magnetic field, the precession of the perihelia of the orbit is determined for our model and compared with the Schwarzschild's one.

In Section 3.3 the geometric character of our model is shown in diagrams of embedding in an Euclidean three space. The fifth dimension is explored also with the help of embedding diagrams. In fact we show in a diagram how the extra dimension shows up near $r=2 M$.

\section{The metric of the 5D magnetic dipole}

The metric that represents the exterior spacetime of a field with magnetic four potential $A=A_{3} d \phi$ and a Schwarzschild-like gravitational potential in the Boyer-Lindquist local coordinates $(r, \theta, \phi, t, y)$, can be written as [7]

$$
\begin{aligned}
\bar{g}= & \frac{1}{I}\left\{\sqrt{I_{o} g_{22}} e^{\tau_{o}^{\prime} \lambda_{d}}\left(\left(1-\frac{2 M}{r}\right)^{-1} d r^{2}+r^{2} d \theta^{2}\right)\left(\frac{r^{2}-2 M r}{\Delta}\right)^{\frac{1}{3}}\right. \\
& \left.+\sqrt{I_{o} g_{22}} r^{2} \sin ^{2} \theta d \phi^{2}-\frac{\left(1-\frac{2 M}{r}\right)}{\sqrt{I_{o} g_{22}}} d t^{2}\right\}+I^{2}\left(A_{3} d \phi+d y\right)^{2} .
\end{aligned}
$$

where

$$
\begin{gathered}
I^{2}=\frac{I_{o}\left(1-\frac{2 M}{r}\right)^{-\frac{2}{3}}}{g_{22}}, \quad g_{22}=\left\{\begin{array}{l}
a e^{q \lambda}+b e^{-q \lambda} \\
b e^{i q \lambda}+\bar{b} e^{-i q \lambda} \\
a \lambda+\frac{1}{I_{o}}
\end{array}, \quad b+\bar{b}=\frac{1}{I_{o}},\right. \\
\lambda=\frac{\tau_{o} M^{2} \cos \theta}{\Delta}, \quad A_{3}=M^{2} \tau_{o}(r-M) \frac{\sin ^{2} \theta}{\Delta}, \\
\Delta=(r-M)^{2}-M^{2} \cos ^{2} \theta,
\end{gathered}
$$

where $\tau_{o}^{\prime}$ is an integration constant and $I_{o}, b, \bar{b}$ and $q$ are constants restricted to $b^{2} q^{2}$ being proportional to $I_{o} . M$ is interpreted as the mass parameter and $\tau_{o}$ is the parameter 
associated with the magnetic field. We observe that the scalar field $I$ appears as a conformal factor in the metric. We interpret the term in brackets \{\} as the spacetime metric, in such a manner that the proper time $\tau$ is given by \{\}$=-c^{2} d \tau^{2}$.

Like other 5D Schwarschild-like solutions, the studied metric does not have event horizons. The curvature is singular for $r=2 M$, therefore, the metric belongs to the soliton class [9]. For $r>>M$ the electromagnetic potential $A=A_{\mu} d x^{\mu}=A_{3} d \phi$ behaves like a magnetic dipole. The metric (2.1) is asymptotically flat for $r>>M$, and flat for $M=0$. When $\tau_{o}=0$ then $A_{3}=0$ and $\lambda=0$ and we have the case without magnetic field. Then the expression in brackets \{\} is like the Schwarzschild metric. In absence of magnetic field we note from (2.2) that $I^{2}$ is a function that increases without bound for $r \rightarrow 2 M$. While for $r>>2 M, I^{2} \rightarrow I_{o}^{2}$, from which we infer that the scalar potential becomes important near $r=2 M$.

This 5D space possess three Killing vectors $\frac{\partial}{\partial y}, \frac{\partial}{\partial t}, \frac{\partial}{\partial \phi}$. As a consequence of the symmetries of the solution, there exist constants of motion which enable us to reduce the problem of finding the geodesics to the analysis of the one-dimensional motion of a particle in an effective potential.

In order to study the geodesic motion in this space, we study the variation of the Lagrangian

$$
\begin{aligned}
\mathcal{L}= & \frac{1}{2 I}\left\{\sqrt{I_{o} g_{22}} e^{\tau_{o} \lambda_{d}}\left(\left(1-\frac{2 M}{r}\right)^{-1}\left(\frac{d r}{d \tau}\right)^{2}+r^{2}\left(\frac{d \theta}{d \tau}\right)^{2}\right)\left(\frac{r^{2}-2 M r}{\Delta}\right)^{\frac{1}{3}}\right. \\
& \left.+\sqrt{I_{o} g_{22}} r^{2} \sin ^{2} \theta\left(\frac{d \phi}{d \tau}\right)^{2}-\frac{\left(1-\frac{2 M}{r}\right)}{\sqrt{I_{o} g_{22}}}\left(\frac{d t}{d \tau}\right)^{2}\right\} \\
& +I^{2} A_{3}^{2}\left(\frac{d \phi}{d \tau}\right)^{2}+2 I^{2} A_{3}\left(\frac{d y}{d \tau}\right)\left(\frac{d \phi}{d \tau}\right)+I^{2}\left(\frac{d y}{d \tau}\right)^{2},
\end{aligned}
$$

where $\tau$ is the proper time in the reference system comoving with the test particle. We restrict to the evolution in the equatorial plane by making $\theta=\frac{\pi}{2}$. The geodesic equation is obtained from the Lagrangian by varying $\mathcal{L}$ with respect to the cyclic coordinates $\theta, t$ and $y$, 


$$
\begin{aligned}
& \frac{\delta \mathcal{L}}{\delta \phi} \rightarrow\left(\frac{\sqrt{I_{o} g_{22}} r^{2}}{I}+I^{2} A_{3}^{2}\right) \frac{d \phi}{d \tau}+I^{2} A_{3} \frac{d y}{d \tau}=B, \\
& \frac{\delta \mathcal{L}}{\delta t} \rightarrow \frac{\left(1-\frac{2 M}{r}\right)}{I \sqrt{I_{o} g_{22}}} \frac{d t}{d \tau}=A, \\
& \frac{\delta \mathcal{L}}{\delta y} \rightarrow I^{2} A_{3} \frac{d \phi}{d \tau}+I^{2} \frac{d y}{d \tau}=\hat{p},
\end{aligned}
$$

$A$ and $B$ are the motion constants associated with conservation of energy and angular momentum, respectively. We have as geodesic equation

$$
\left(\frac{d r}{d \tau}\right)^{2}+e^{\left(\frac{-\tau_{o}^{2} \tau_{o}^{\circ}}{8(r-1)^{4}}\right)}\left\{\left(\frac{I^{2}\left(B-\hat{p} A_{3}\right)^{2}}{I_{o} g_{22} r^{2}}+\frac{c^{2}}{\sqrt{I_{o} g_{22}}}\right)\left(1-\frac{2 M}{r}\right)-A^{2} I^{2}\right\}\left(\frac{\Delta}{r^{2}-2 M r}\right)^{\frac{1}{3}}=0,
$$

From the previous equation we have for the effective potential the expression

$$
V=\exp \left(\frac{-\tau_{o}^{2} \tau_{o}^{\prime}}{8(r-1)^{4}}\right)\left(\frac{I^{2}\left(B-\hat{p} A_{3}\right)^{2}}{I_{o} g_{22} r^{2}}+\frac{c^{2}}{\sqrt{I_{o} g_{22}}}\right)\left(1-\frac{2 M}{r}\right)\left(\frac{\Delta}{r^{2}-2 M r}\right)^{\frac{1}{3}}
$$

\section{Testing the model}

\subsection{The effective potential for a test particle}

To deduce the qualitative features of the orbits we analyze the behaviour of the effective potential (2.6) for a test particle with angular momentum per mass $l$ and energy $E_{o}$ (at infinity) in presence of our $5 \mathrm{D}$ massive magnetic dipole with mass $\mathrm{M}$ and dipole parameter $\tau_{o}$. Substituting $A_{3}$ from (2.3) with $\theta=\frac{\pi}{2}, \gamma=\hat{p} \tau_{o}$ and $l=\frac{B}{M}$ we arrive to

$$
V=\exp \left(\frac{-\tau_{o}^{2} \tau_{o}^{\prime}}{8(r-1)^{4}}\right)\left[\left(\frac{l}{r}-\frac{\gamma}{(r-1) r}\right)^{2}+\left(1-\frac{2}{r}\right)^{\frac{2}{3}}\right]\left(1-\frac{2}{r}+\frac{1}{r^{2}}\right)^{\frac{1}{3}},
$$

where in order to make the plotting we have scaled $\frac{r}{M} \rightarrow r$ and $c=1$. For the case in absence of magnetic field we put in (3.1), $\gamma=0\left(\tau_{o}=0\right)$.

It can be seen that the last term in $(2.5)$ is proportional to $\left(1-\frac{2 M}{r}\right)^{-\frac{2}{3}}\left(\frac{\Delta}{r^{2}-2 M r}\right)^{\frac{1}{3}}$. In order to perform a comparison with Schwarzschild's solution, we have taken in expression (3.1) the energy $E=E_{o}\left(1-\frac{2 M}{r}\right)^{-1}\left(1-\frac{2 M}{r}+\frac{M^{2} \sin ^{2} \theta}{r^{2}}\right)^{\frac{1}{3}}$ to zeroth order. It invalids our approximation for the region $r \rightarrow 2 M$ because at this points the energy diverges. 
In Fig. 1 we can visualize the principal features of typical orbits and the effects of varying the magnetic dipole parameter $\gamma$. The quantity plotted is $\sqrt{1+V}$.

Fig. 1.a. shows, in absence of magnetic dipole $\left(\tau_{o}=0\right)$, the effect on the effective potential of the variation of the angular momentum $l$ of the test particle. We take as reference for the values of the angular momentum of the test particle the study by Misner, Wheeler and Thorne (see Ch 25, Ref [10]). Fig. 1.a. can be compared with the effective potential corresponding to Schwarzschild's solution (Fig. 25.2 in Ref [10]). It reveals the following features: stable circular orbits are possible for the same values of the angular momentum $l$, but the radious value of the stable orbits are greater for the $5 \mathrm{D}$ model than for Schwarzschild's solution. For the 5D model the last stable circular orbit corresponds to $l=2.03$ while for Schwarzschild's solution this value is $l=3.464$.

Qualitatively the effect is the same as in Schwarzschild's geometry: the minimum (stable circular orbits) in the 5D model is "dragged into the hole", i. e. it is moved inwards for $l$ decreasing. However, in the 5D model there are no a maximum (unstable circular orbits) and for the region near $r=2 M$, the effect of the scalar field appears as a "core" which rejects particles which in Schwarzchild's geometry would penetrate the hole. In Fig. 1.b. both Schwarzschild and 5D effective potentials are displayed for $l=4$.

In Fig. 1.c. it is shown the energy $E$ which we have taken to zeroth order and the effective potential $V$ corresponding to $l=4.33$. It occurs that the term $A^{2} I^{2}$ which we associate with the energy of the test particle, is not constant and in fact it increases without bound near horizon. We can attribute this effect to the interaction of the particle with the massless scalar field making the particle augment its velocity in such a way that it can not be trapped by the body. The effect of this energy would be like an impenetrable core for a positive energy (neglecting the quantum tunnel effect). It maybe can fit with a fluid like interpretation of the fifth dimension. In Fig. 1.d. it is shown the radial velocity of the particle, $\dot{r}=(E-V)^{\frac{1}{2}}$, this velocity augments as the particle gets nearer the body and when the particle is about $r=2 M$ it suddenly increases without bound.

Turning now to the magnetic case in (3.1), Fig. 1.e. shows the dependence of the effective potential $V$ with respect to the variation of the magnetic dipole parameter $\tau_{o}$. In this case the value of $\tau_{o}$ influences in a significative way the form of the effective potential. The effect of the magnetic dipole is to low the potential barrier. This means that as $\tau_{o}$ 
increases, the energy for a charged particle to be captured decreases. Also, the effective potential is sensitive to changes of the parameter $\gamma$. From its definition as $\gamma=\hat{p} \tau_{o}$, where $\hat{p}$ is the fifth-momentum of the test particle, the value of $\gamma$ can not be prescribed in advance, but rather it needs to be adjusted according to observational data. In Fig. 1.f. it is compared the effective potential $V$ for a magnetic dipole with $\gamma=10$ and $\gamma=50$, $\tau_{o}^{2} \tau_{o}^{\prime}=1$, with the one in absence of magnetic field.

In [11] a solution for a massive magnetic dipole in four dimensions is studied. Solution in [11] is singular in polar caps and therefore test particles can never reach polar caps. For the solution analyzed here this effect does not exist. It can be seen from the expression for $A_{3}$ in (2.3) that the magnetic dipole vanishes at polar caps $(\sin \theta=0)$.

In the previous plots, it is manifest that the capture into the body can occur whenever the energy of the test particle exceeds the effective potential $V$. However if we take into account the term $E$ then no particle can be captured by the body. It can be considered as an effect of the scalar field surrounding the body.

\subsection{Precession of the perihelia of the orbit.}

In order to determine the prediction of our model for the precession of the perihelia of the orbit we use the equation for $r=r(\phi)$. We perform the change of variable

$$
r=\frac{1}{u}, \quad \frac{d r}{d \tau}=\frac{d r}{d \phi} \frac{d \phi}{d \tau}=u^{\prime} \frac{d \phi}{d \tau} .
$$

Substituting in $\left\{\frac{d s}{d \tau}\right\}^{2}=-c^{2}$, the geodesic equation is

$$
u^{\prime 2}+e^{-\tau_{o}^{\prime} \lambda_{d}}\left[u^{2}(1-2 M u)-\frac{A^{2} I_{o} g_{22}}{\left(B-p A_{3}\right)^{2}}+\frac{c^{2}(1-2 M u) \sqrt{I_{o} g_{22}}}{I^{2}\left(B-p A_{3}\right)^{2}}\right] \frac{(1-M u)^{\frac{2}{3}}}{(1-2 M u)^{\frac{1}{3}}}=0
$$

where $A$ and $B$ are the constants of motion and

$$
\lambda_{d}=\frac{\tau_{o}^{2} M^{4}}{8(r-M)^{4}}, \quad A_{3}=\frac{M^{2} \tau_{o}}{r-M}
$$

When (3.3) is considered in the form $u^{\prime 2}+V=0, V$ is the expression in square brackets. Neglecting terms in $u^{3}$ we have,

$$
u^{\prime \prime}+\frac{\partial V}{\partial u}=0
$$


with

$$
\frac{\partial V}{\partial u}=\frac{1}{k}-\omega^{2} u+\omega_{1} u^{2}
$$

with the frequencies $\omega, \omega_{1}$ defined by

$$
\begin{gathered}
\omega^{2}=-\frac{3 \gamma_{1}+2 M}{k}+1+\alpha\left(\frac{53 M}{15}-\gamma_{1}\right)-\frac{A^{2} M^{2}}{3 B^{2}} \\
\omega_{1}=\frac{M^{2}+3\left(M+\gamma_{1}\right)\left(M+2 \gamma_{1}\right)}{k}+3 M-\alpha\left(\frac{182 M^{2}}{45}+\frac{113 M \gamma_{1}}{15}-3 \gamma_{1}^{2}\right)-\frac{85 A^{2} M^{3}}{27 B^{2}}
\end{gathered}
$$

and

$$
k=\frac{1}{\alpha+\gamma_{1}\left(\frac{A^{2}}{B^{2}}-\frac{3 \alpha}{5 M}\right)}, \quad \alpha=\frac{5 c^{2} M}{3 I_{o}^{2} B^{2}}, \quad \gamma_{1}=\frac{\hat{p} \tau_{o}}{B},
$$

Now we have to solve (3.4) in order to comprehend the geodesic motion of a test particle. Solving it in a first order approximation we obtain

$$
u_{o}=\frac{1}{k}\left(\frac{1}{\omega^{2}}+\epsilon \cos \omega \phi\right)
$$

with $\epsilon$ being the excentricity of the orbit. We then replace this result in $u^{2}$ in $(3.4)-(3.5)$ and solving it we obtain the solution

$$
u_{1}=\frac{1}{k \omega^{2}}\left[1+\epsilon \cos \left(\frac{\omega_{1}}{k \omega}-\omega\right) \phi\right]
$$

The expressions for the frequencies from (3.6) with $\tau_{o}=0$ (absence of magnetic field) reduce to

$$
\omega^{2}=1+\frac{23}{15} \alpha M-\frac{A^{2} M^{2}}{3 B^{2}}, \quad \omega_{1}=3 M-\frac{2}{45} M^{2} \alpha-\frac{85 A^{2} M^{3}}{27 B^{2}},
$$

From the previous expression the precession of the perihelia can be calculated as

$$
\Delta \phi=2 \pi\left(1-\frac{k \omega}{\omega_{1}-k \omega^{2}}\right) r a d
$$

If, for instance, we try for Mercury, we obtain a precession of $43.01^{\prime \prime}$ (sec/century). The approximation is in good agreement with the observed data as well as with the Schwarzschild's prediction. It is easy to show that the constants in the final expression (3.9) involve only the mass of the Sun $M$ and the constant $k=\alpha^{-1}=\left(1-\epsilon^{2}\right) a$, with $a$ being the semimajor 
axis and $\epsilon$ the excentricity of the eliptic orbit of Mercury. So, at this point we haven't made any assumption about the magnitude of the fifth dimension.

\subsection{Embedding}

In order to visualize the geometry of the space around this 5D Schwarzschild-like body in a convenient manner, we show the corresponding embedding diagrams. For the line element (2.1) in absence of magnetism,

$$
\begin{gathered}
d s^{2}=\frac{1}{I_{o}}\left(1-\frac{2 M}{r}\right)^{\frac{1}{3}}\left\{\left(\frac{1-\frac{2 M}{r}}{1-\frac{2 M}{r}+\frac{M^{2} \sin ^{2} \theta}{r^{2}}}\right)^{\frac{1}{3}}\left(\frac{d r^{2}}{1-\frac{2 M}{r}}+r^{2} d \theta^{2}\right)+r^{2} \sin ^{2} \theta-\left(1-\frac{2 M}{r}\right) d t^{2}\right\}+ \\
\frac{I_{o}^{2} d y^{2}}{\left(1-\frac{2 M}{r}\right)^{\frac{2}{3}}} .
\end{gathered}
$$

The geometry that results of making $t=$ const., $\theta=\frac{\pi}{2}$ and $y=$ const. is the line element

$$
d s^{2}=\frac{1}{I_{o}}\left(1-\frac{M}{r}\right)^{-\frac{2}{3}}\left(1-\frac{2 M}{r}\right)^{-\frac{1}{3}} d r^{2}+\frac{r^{2}}{I_{o}}\left(1-\frac{2 M}{r}\right)^{\frac{1}{3}} d \phi^{2} .
$$

We proceed to embed it in the flat geometry of an Euclidean three dimensional manifold. We take the Euclidean three space in cylindrical coordinates $(z, r, \phi)$ and we identify the $z$ and $\phi$ of the slice obtained from (3.11) with the $z$ and the $\phi$ of the Euclidean 3-space. Then the line element in the two cases are to be identical (see Ref [10], Ch. 23). The line element

$$
d s^{2}=\left[1+\left(\frac{d z(r)}{d r}\right)^{2}\right] d r^{2}+r^{2} d \phi^{2}
$$

on the two-dimensional locus in the 3-geometry is identified with

$$
d s^{2}=f(R) d R^{2}+R^{2} d \phi^{2}
$$

from (3.12) in our body, where

$$
f(R)=\frac{\gamma_{o}^{6} R^{8}}{\left(r^{3}-\frac{5}{3} r^{2}\right)^{2}\left(r^{3}-r^{2}\right)^{\frac{2}{3}}}, \quad R=r \gamma_{o}^{-\frac{1}{2}}\left(1-\frac{2}{r}\right)^{\frac{1}{6}}, \quad \gamma_{o}=\frac{I_{o}}{M^{2}}
$$


here we have scaled $r \rightarrow \frac{r}{M}$. Comparing (3.13) and (3.14) it is obtained the embedding formula

$$
1+\left(\frac{d z}{d R}\right)^{2}=f(R), \quad d z=\sqrt{\{f(R)-1\}} d R .
$$

The embedded surface is a segment of a paraboloid of revolution. It is shown in Fig. 2.c. $R$ is rescaling the radial dimension $r$. The location of the singularity now depends on the value of the constant $\gamma_{o}$, associated with the massless scalar field (see (3.15)). Making $\phi$ to vary in $(0,2 \pi)$ we obtain Fig. 2.a. Fig. 2.b. shows the corresponding embedding for Schwarzschild metric. The embedding diagram in Fig.2.a. resembles Schwarzschild's geometry with a "wormhole" that could connect two distinct asymptotically flat universes.

From Figs. 2.c. and 2.b. we can compare $z(R)$ from $5 \mathrm{D}$ with $z(r)$ for Schwarzschild's solution. 2.c. is plotted for $\gamma_{o}=1.3$.

We now turn to the embedding of the fifth dimension. The exploration we perform of the fifth dimension here is in the line of Ref [5], i. e. as a geometric property which corresponds to an inner symmetry, rather than the usual approach which takes its fourthdimensional interpretation as a massless scalar field. Performing a similar identification for the fifth dimension $I$ instead of $r$ and $y$ instead of $\phi$, taking $y$ as the 5D angle variable, we have to identify the 3-geometry in (3.13) with the slice obtained from the line element (3.11) for $t=$ const., $\phi=$ const. and $\theta=\frac{\pi}{2}$,

$$
d s^{2}=\frac{36 I^{7} d I^{2}}{\gamma_{o}\left(I^{3}-1\right)^{4}\left[I^{3}+\frac{1}{4}\left(I^{3}-1\right)^{2}\right]^{\frac{1}{3}}}+I^{2} d y^{2}=g(I) d I^{2}+I^{2} d y^{2},
$$

From (3.13) and (3.17) we obtain the embedding formula,

$$
d z=\sqrt{\{g(I)-1\}} d I,
$$

where we have scaled to $\frac{I}{I_{o}}$. A real embedding is obtained for values of $\gamma_{o}$ from $10^{-6}$ and lower. Since this behaviour is depending on values of the constant $\gamma_{o}=\frac{I_{o}}{M^{2}}$ it seems that we can not have the two embeddings simultaneously, i. e. we must choose which dimension we want to see, but we can not see both at the same instant when we approach $r=2 M$. This could be an artificial effect due to the form in which we have to define $R$ for the embedding. From (3.15) it can be seen that only for $\gamma_{o}>1$ (when $R>r$ ) we can obtain for $R$ a real embedding. This embedding is shown in Fig. 3. 
Fig. 4. is a draw of how the fifth dimension would show up near $r=2 M$. This makes sense when we think of $I$ as the fifth dimensional radious, i. e. a dimension which is contracted and shows up near $r=2 M$. From (2.2), $I_{o}$ is the value of this radious at infinity in such a manner that when one approaches $r=2 M$, this radious increases without limit.

We also studied the redshift and null geodesics . They turn out to be the same as for

Schwarzschild's solution as can be seen from the equation $\left\{\frac{d s^{2}}{d \tau^{2}}\right\}=0$ from which the same Schwarzschild's equation for geodesics is obtained.

\section{Conclusions}

We have analyzed the behaviour of a test particle moving in the effective potential of a 5D Schwarzschild-like model interpreted as a massive magnetic dipole coupled with a massless scalar field. The model behaves in agreement with Schwarzschild's solution for regions not near $r=2 M$, as shows the prediction for the precession of the perihelia of Mercury. In the neighborhood of $r=2 M$, it changes dramatically and fifth-dimensional effects show up. We have discussed both interpretations, as a massless scalar field and as a fifth-dimensional geometric property of space near the singularity $r=2 M$. The constant $\gamma_{o}=\frac{I_{o}}{M^{2}}$ deserves further attention in order to clarify its value according to real data.

The study of a solution involve a lot of interesting aspects besides the ones analyzed here. We can point out, for instance, the study of the 4D energy momentum tensor which corresponds to this solution; the study of the behaviour of test particles near polar caps and of future interest is the generalization of this solution to the stationary case in order to model in a more realistic way a rotating astrophysical object. It is also convenient a deeper investigation on the relationship between this model and the string theory for low energies.

\section{Acknowledgements}

This work is partially supported by CONACyT (México). 


\section{References}

[1] V. S. Manko and N. R. Sibgatullin, J. Math. Phys. 34, 170 (1993).

Ts. I. Gutsunaev and V. S. Manko, Gen. Rel. Grav. 22, 799 (1988).

[2] M. J. Duff, B. F. Nilsson and C. V. Pope Phys. Rep. 130, 1 (1986).

R. N. Mohapatra Unification and Supersymmetry Springer- Verlag (1992)

T. Matos and A. Nieto Rev. Mex. Fis. Suplement No. 2 (1993)

[3] P.S. Wesson and J. Ponce de León, J. Math. Phys. 33, 3883 (1992).

P. S. Wesson, Phys. Lett. B276, 299 (1992).

[4] P. S. Wesson, Gen. Rel. and Grav. 22, 707 (1990).

[5] A. Davidson and D. A. Owen, Phys. Lett. B155, 247 (1985).

[6] T. Matos, "5D Axisymmetric Stationary Solutions as Harmonic Maps". J. Math. Phys. 35 (1994).

[7] T. Matos. Phys. Rev. D48, (1994).

[8] D. Garfinkle, G. T. Horowitz and A. Strominger Phys. Rev. D43, 3140 (1991).

[9] H. Liu, Gen. Rel. Grav. 23, 759, (1991).

T. Dereli, Phys. Lett. B161, 307 (1985).

D. J. Gross and M. J. Perry, Nuclear Phys. B226, 29 (1983).

[10] Ch. Misner, K. S. Thorne, J. A. Wheeler Gravitation Ed. Freeman and Co. (1973).

[11] D. Vokrouhlicky and V. Karas, Gen. Rel. Grav. 22, 1033 (1990) 


\section{Figure Captions}

Fig. 1 The principal features of typical orbits and the effects on the effective potential of varying the magnetic dipole parameter $\tau_{o}$ are shown. The quantity plotted is $\sqrt{1+V}$.

a). The effective potential's variations for distinct values of the angular momentum $l$ of the test particle, $l=4.33,4,3.75,3.464,2.5$.

b). Here are displayed both Schwarzschild's and 5D effective potentials for $l=3.75$.

c). Energy $E=E_{o}\left(1-\frac{2}{r}\right)^{-1}\left(1-\frac{2 M}{r}+\frac{M^{2} \sin ^{2} \theta}{r^{2}}\right)^{\frac{1}{3}}$ and the effective potential $V$ for $l=4.33$ are plotted.

d). It is shown the variation of the radial velocity of the particle, $\dot{r}=(E-V)^{\frac{1}{2}}$. It increases without bound near $r=2 M$.

e). The effect on the effective potential $V$ of the magnetic dipole parameter variation is displayed for $\gamma=1$ and $\tau_{o}^{2} \tau_{o}^{\prime}=10,50,100$.

f). The cases with magnetic dipole for $\tau_{o}^{2} \tau_{o}$, $=1$ and $\gamma=10,50$ are compared with the case in absence of magnetism.

Fig. 2 Embedding in an Euclidean 3-space is displayed.

a). The paraboloid of revolution in (c) is rotated and reflected in the equatorial plane to generate the "wormhole".

b). Here it is plotted the embedding for Schwarzschild, $z(r)=\int \frac{d r}{\sqrt{\frac{r}{2}-1}}$.

c). Embedding for 5D metric. The points are the result of the numerical integration $z(R)=\int \sqrt{f(R)-1} d R$. With $f(R)$ from (3.15).

Fig. 3 The embedding for the fifth dimension $I$ is shown. In the lower part is shown the paraboloid of revolution which results from integrating $z(I)=\int \sqrt{g(I)-1} d I$, $g(I)$ given in (3.17). Above, the same paraboloid has been rotated and reflected in an equatorial plane.

Fig. 4 This is a draw which shows how the radious of the fifth dimension $I$ changes as a function of $r$. According to (2.2), it increases from a small value at infinity to an infinite radious near $r=2 M$. 